\title{
Real-Time Path Integral Simulation of Exciton-Vibration Dynamics in Light Harvesting Bacteriochlorophyll Aggregates
}

Sohang Kundu ${ }^{1}$ and Nancy Makri ${ }^{1,2 *}$

${ }^{1}$ Department of Chemistry, University of Illinois, Urbana, Illinois 61801

${ }^{2}$ Department of Physics, University of Illinois, Urbana, Illinois 61801

${ }^{*}$ Corresponding Author.

\section{Supporting Information}

\section{Hamiltonian}

The Frenkel exciton Hamiltonian ${ }^{1}$ for an aggregate of $n \mathrm{BChl}$ units is a sum of single monomer terms and intermonomer electronic interactions,

$$
\hat{H}=\sum_{\alpha=1}^{n} \hat{H}^{\alpha}+\sum_{\alpha=1}^{n-1} J^{\alpha, \alpha+1}\left(\left|0^{\alpha} 1^{\alpha+1}\right\rangle\left\langle 1^{\alpha} 0^{\alpha+1}|+| 1^{\alpha} 0^{\alpha+1}\right\rangle\left\langle 0^{\alpha} 1^{\alpha+1}\right|\right)
$$

where

$$
\hat{H}^{\alpha}=\left(\varepsilon_{0}^{\alpha}+\hat{h}_{0, \text { vib }}^{\alpha}\right)\left|0^{\alpha}\right\rangle\left\langle 0^{\alpha}\left|+\left(\varepsilon_{1}^{\alpha}+\hat{h}_{1, \text { vib }}^{\alpha}\right)\right| 1^{\alpha}\right\rangle\left\langle 1^{\alpha}\right|
$$

Here $\left|0^{\alpha}\right\rangle$ and $\left|1^{\alpha}\right\rangle$ are the ground and excited electronic states of pigment $\alpha, \varepsilon_{0}^{\alpha}$ and $\varepsilon_{1}^{\alpha}$ are their energies, $J^{\alpha, \alpha+1}$ is the electronic coupling constant between adjacent monomers. If the aggregate has a circular topology, Eq. (1) contains an additional term that couples units $n$ and 1. The vibrational Hamiltonians of the ground and excited electronic states are given by

$$
\hat{h}_{0, \mathrm{vib}}^{\alpha}=\sum_{j=1}^{v} \frac{\left(\hat{p}_{j}^{\alpha}\right)^{2}}{2 m}+\frac{1}{2} m \omega_{j}^{2}\left(\hat{q}_{j}^{\alpha}\right)^{2}, \quad \hat{h}_{1, \mathrm{vib}}^{\alpha}=\sum_{j=1}^{v} \frac{\left(\hat{p}_{j}^{\alpha}\right)^{2}}{2 m}+\frac{1}{2} m \omega_{j}^{2}\left(\hat{q}_{j}^{\alpha}-\frac{c_{j} s_{1}}{m \omega_{j}^{2}}\right)^{2}
$$

where $q_{j}^{\alpha}, p_{j}^{\alpha}$ are the $v$ normal mode coordinates and momenta (i.e. $m=1$ ) of monomer $\alpha$, and $s_{1}=2$ (to conform with the common spin-boson form). The displacement coordinates $d_{j}=c_{j} s_{1} / m \omega_{j}^{2}$ have the same value for all (identical) BChl monomers and are related to the Huang-Rhys factors $S_{j}$ through the expression $S_{j}=m \omega_{j} d_{j}^{2} / 2 \hbar$, which implies $c_{j}=\sqrt{\frac{1}{2} m \omega_{j}^{3} \hbar S_{j}}$.

\section{Initial Condition}

The initial density operator at $t=0$ is a product of electronic and vibrational components,

$$
\hat{\rho}(0)=\prod_{\alpha=1}^{n} \hat{\rho}_{\mathrm{el}}^{\alpha}(0) \hat{\rho}_{\mathrm{vib}}^{\alpha}(0)
$$


It is assumed that the initial excitation is localized on a single pigment $\alpha_{0}$, i.e., $\hat{\rho}_{\mathrm{el}}^{\alpha_{0}}(0)=\left|1^{\alpha_{0}}\right\rangle\left\langle 1^{\alpha_{0}}\right|$, $\hat{\rho}_{\mathrm{el}}^{\alpha}(0)=\left|0^{\alpha}\right\rangle\left\langle 0^{\alpha}\right|$ for $\alpha \neq \alpha_{0}$. In accordance with the Franck-Condon principle, all vibrational modes at $t=0$ are equilibrated to the electronic ground state of each monomer, $\hat{\rho}_{\text {vib }}^{\alpha}(0)=e^{-\beta h_{0, v i b}^{\alpha}} / \operatorname{Tr} e^{-\beta \hat{h}_{0, v i b}^{\alpha}}$.

\section{Observable}

The RDM $\rho_{i j}^{\alpha}$ of monomer $\alpha$ is given by the partial trace of the time-dependent density matrix with respect to the vibrational states of all units and the electronic states of all monomers besides $\alpha$. Since the Hamiltonian does not allow multiple excitations to be created from a single excited $\mathrm{BChl}$ unit, if the excitation is found on pigment $\alpha$, all other pigments must be in the ground state. Thus the trace with respect to electronic states may be dropped, and the RDM is given by

$$
\rho_{\alpha \beta}(t)=\operatorname{Tr}_{\text {vib }}\left\langle 0^{1} 0^{2} \cdots 1^{\alpha} \cdots 0^{n}\left|e^{-i \hat{H} t / \hbar} \hat{\rho}(0) e^{i \hat{H} t / \hbar}\right| 0^{1} 0^{2} \cdots 1^{\beta} \cdots 0^{n}\right\rangle
$$

The excited state population (i.e. the survival probability) $P_{\alpha}(t)$ of monomer $\alpha$ is given by

$$
P_{\alpha}(t)=\rho_{\alpha \alpha}(t)
$$

\section{Modular Path Integral}

The modular path integral (MPI) methodology $y^{2,3}$ takes advantage of the locality of interactions to propagate the density matrix by sequentially linking the quantum paths of a monomer to those of the adjacent unit, thus achieving linear scaling with system size. A difficulty arises because of the absence of a single-particle product basis that diagonalizes the coupling term for the exciton Hamiltonian. We recently showed that the apparent doubling of path integral variables can be overcome ${ }^{4}$ and introduced a factorization of the linking process ${ }^{5}$ that leads to a dramatic acceleration. Further, the MPI approach allows a fully quantum mechanical treatment of any number of normal mode vibrational degrees of freedom, at zero or finite temperature, essentially at no additional cost. The vibrations modify the path amplitudes via analytical expressions that depend on the frequency and coupling coefficient of each mode, as well as the temperature. ${ }^{6}$ This flexibility allows the inclusion of all normal mode vibrations with the precise values of Huang-Rhys factors obtained from experimental spectra.

\section{Parameters}

The electronic and vibrational parameters for BChl aggregates used in the simulations are for the $\mathrm{B} 850$ ring of the LH2 complex found in purple bacteria. The electronic couplings and excitation energies were obtained from electronic structure calculations. ${ }^{7,8}$ The Huang-Rhys parameters and frequencies for the 50 most strongly coupled vibrational modes were obtained from spectroscopic data. ${ }^{9}$

\section{References}

1. Frenkel, J., On the transfomation of light into heat in solids. Phys. Rev. 1931, 37, 17.

2. Makri, N., Modular path integral: Quantum dynamics via sequential necklace linking. J. Chem. Phys. 2018, 148, 101101. 
3. Makri, N., Modular path integral methodology for real-time quantum dynamics. J. Chem. Phys. 2018, 149, 214108.

4. Kundu, S.; Makri, N., Modular path integral for discrete systems with non-diagonal couplings. $J$. Chem. Phys. 2019, 151, 074110.

5. Kundu, S.; Makri, N., Efficient matrix factorization of the modular path integral for extended systems. Mol. Phys. 2020.

6. Kundu, S.; Makri, N., Modular path integral for finite-temperature dynamics of extended systems with intramolecular vibrations. J. Chem. Phys. 2020, 153, 044124.

7. Tretiak, S.; Middleton, C.; Chernyak, V.; Mukamel, S., Exciton Hamiltonian for the bacteriochlorophyll system in the LH2 antenna complex of purple bacteria. J. Phys. Chem. B 2000, 104, 4519-4528.

8. Strümpfer, J.; Schulten, K., The effect of correlated bath fluctuations on exciton transfer. The Journal of Chemical Physics 2011, 134, 095102.

9. Rätsep, M.; Cai, Z.-L.; Reimers, J. R.; Freiberg, A., Demonstration and interpretation of significant asymmetry in the low-resolution and high-resolution Qy fluorescence and absorption spectra of bacteriochlorophyll a. J. Chem. Phys. 2011, 134, 024506. 